\begin{tabular}{|c|c|c|c|c|}
\hline & 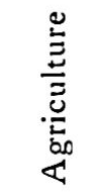 & 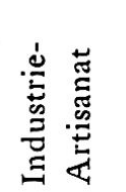 & 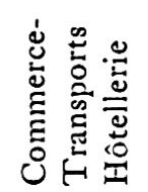 & 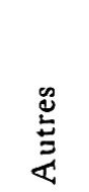 \\
\hline L'Abbaye & 53 & 337 & 53 & $\begin{array}{r}51 \\
260\end{array}$ \\
\hline $\begin{array}{l}\text { Le Chenit } \\
\text { Le Lieu }\end{array}$ & $\begin{array}{r}141 \\
74\end{array}$ & $\begin{array}{r}1584 \\
294\end{array}$ & $\begin{array}{r}192 \\
55\end{array}$ & $\begin{array}{r}260 \\
60\end{array}$ \\
\hline La Vallée & 268 & 2215 & 300 & 371 \\
\hline
\end{tabular}

\title{
DER STRUKTURWANDEL DER ALPWIRTSCHAFT IM LUFTBILD (AM BEISPIEL DES DISCHMATALES)
}

\author{
HAROLD HAEFNER
}

\section{DAS PROBLEM}

Die Strukturwandlungen der Alpwirtschaft und die Veränderungen im alpinen Landschaftsbild in jüngster Zeit sind bekannt und mehrfach beschrieben worden ( $\mathrm{Pa}$ schinger 1962, Suter 1963, Gutersohn 1963, 1964 usw.). Es handelt sich vorwiegend um Extensivierungsvorgänge, um Bevölkerungsabwanderungen, Änderungen in der Wirtschaftsform (Aufgabe der jahreszeitlichen Wanderungen, Aufgabe der höchst- und entferntest gelegenen Alpen, Wechsel von Kuh- in Jungvieh- oder Schafalpen usw.). Andererseits wird versucht, durch tiefgreifende Sanierungen geeigneter Objekte rationellere Nutzung und Qualitätsverbesserung zu erreichen (Melioration, Wegerschließung, Bau von Transportbahnen und Milchpipelines, Stallsanierung, Weideverbesserung, Erstellung von Verschlauchungsanlagen usw.). Die Extensivierungsvorgänge sind bedeutend und nehmen in den letzten Jahren an Umfang eher noch zu. Die positiven Strukturänderungen dagegen sind viel geringer und werden nur zögernd und in unzulänglicher Weise an die Hand genommen.

Der allgemeine Trend ist bekannt. Über das eigentliche Ausmaß der Vorgänge aber macht man sich kaum eine Vorstellung, da bisher nie versucht wurde, diese Phänomene in ihrer räumlichen Verbreitung und zeitlichen Abfolge exakt festzuhalten. Gerade derartige Untersuchungen sind aber notwendig, um uns ein klares Bild über die Bedeutung dieser Vorgänge und die Dringlichkeit zu energischer Hilfe vor Augen zu führen. Die kartographische Erfassung dieser Strukturänderungen verursacht auch bedeutende Schwierigkeiten. Hier kann nun das Luftbild sinnvoll eingesetzt werden und außerordentlich wertvolle Dienste leisten. Oft wird es sogar die einzige Quelle sein, die uns genauere Angaben über diese Veränderungen in der Wirtschaftslandschaft zu geben vermag. Die sich hier bietenden Möglichkeiten und die anzuwendenden Arbeitsmethoden sollen im folgenden für die Belange der Alpwirtschaft besprochen und anhand eines praktischen kleinen Beispiels erläutert werden.

\section{DIE MÖGLICHKEITEN DER LUFTBILDINTERPRETATION}

Im Luftbild besitzt die geographische Forschung ein wertvolles kulturhistorisches Dokument, das einen einmaligen und nicht wiederholbaren Zustand der Landschaft zu 
einem genau fixierten Zeitpunkt festhält. Je älter, desto kostbarer sind darum die Bilder, und es ist von höchster Wichtigkeit, alle sorgfältig zu sammeln und aufzubewahren.

Gerade zur Erfassung der Veränderungen der Kulturlandschaft erweist es sich von besonderem Vorteil, wenn ältere Bildflüge zur Verfügung stehen. Mit Hilfe eines Bildvergleichs lassen sich die Strukturwandlungen am besten ermitteln. Sind sogar mehrere Bildserien vorhanden, so läßt sich auch der zeitliche Ablauf rekonstruieren.

Die Interpretation alter Bildflüge ist allerdings nicht immer einfach, da sie meistens von eher geringerer photographischer Qualität sind und die Möglichkeit der Feldkontrolle dahinfällt. Somit können Unsicherheiten, die bei der Bildauswertung auftreten, nicht mehr geklärt werden. Die zuverläßigste und erfolgversprechendste Methode der Interpretation ist die mit Hilfe eines Schlüssels. Dieser wird aus neuen Luftbildern vom gleichen Gebiet, die in Filmart, Maßstab und Jahreszeit denjenigen des alten Fluges möglichst entsprechen sollen, zusammengestellt und im Feld auf seine Richtigkeit überprüft. Nur auf diesem Weg kann eine optimale Auswertung alter Bildflüge gesichert werden.

Schon ein einzelnes Luftbild erlaubt uns Aussagen in verschiedenster Hinsicht. Es vermittelt nicht nur den gegenwärtigen Zustand einer Landschaft, sondern enthält auch die Spuren früherer Zustände und gibt Anhaltspunkte für die Weiterentwicklung und die sinnvolle Planung.

Für die Alpwirtschaft und die alpine Kulturlandschaft lassen sich dem Luftbild folgende wesentliche Erkenntnisse entnehmen:

\section{Erfassung des gegenwärtigen Zustandes:}

(Nähere Angaben über Interpretationskriterien, siehe: Steiner und Haefner 1963, Haefner 1963 a. u. b.)

Art der Vegetationsbedeckung - Art der Landnutzung - Zustand des Waldes (Baumarten, Alter, Dichte, Schutzfunktionen usw.) - Zustand von Wiesen und Weiden (Dichte des Graswuchses, Bodenfeuchtigkeit, Be- oder Entwässerung, Lesesteinhaufen usw.) - Unterscheidung einzelner Wiesentypen - Gefährdung durch Lawinen - Beschaffenheit der Schneedecke - Ablauf der Ausaperung im Frühjahr - Gebäudetypisierung - Zustand der Erschließung (Wegnetz, Transportbahnen usw.) - Gliederung in einzelne Betriebsstufen evtl. Betriebseinheiten.

\section{Erfassung frïherer Zustände: (Haefner 1963 b)}

Hauswüstungen - Flurwüstungen - Aufgegebene Mähwiesen - Altes Wegnetz - Alte Beoder Entwässerungssysteme - Alte Fluß-oder Bachläufe.

\section{Planungsunterlagen: (Ramser 1951, Steiner und Haefner 1963)}

Die gründliche Landschaftsanalyse gibt eine vertiefte Einsicht, wie und wo mit Verbesserungen eingesetzt werden kann und soll. Stellen lassen sich festlegen, wo gezielte Feldarbeit einzusetzen hat. Die Alpsanierung kann so ganz allgemein erleichtert und beschleunigt werden, z. B. im Hinblick auf:

Lawinenverbauungen - Wildbachverbauungen - Gebäudeneubauten - Geländeerschließung - Betriebstechnische Reorganisationen - Ausscheidung von Wald und Weide - Aufforstung.

Umgekehrt darf man nicht erwarten, stets all diese Angaben demselben Luftbild entnehmen zu können. Jahreszeit, Maßstab und Tageszeit bilden hier die limitierenden Faktoren. Kenntnisse über diese Zusammenhänge müssen vorausgesetzt werden um zu wissen, was man von einem bestimmten Luftbild erwarten und ihm entnehmen kann und was nicht. Zudem sind phänologische Daten und Angaben über den Verlauf des landwirtschaftlichen Jahres vom betreffenden Arbeitsgebiet unerläßlich. Besonders im Gebirge sind diese Anhaltspunkte von Bedeutung, da sie gegenüber den uns gewohnten Daten erheblich differieren.

Für alpwirtschaftliche Untersuchungen auf Luftbildern eignen sich vor allem zwei Jahreszeiten.

1. Die einsetzende Vegetationsentwicklung (Mai-Juni, je nach Höhenlage) mit sehr guten Grautondifferenzen zwischen den einzelnen Vegetationseinheiten, Baum- und Gebüscharten und Wiesentypen. 


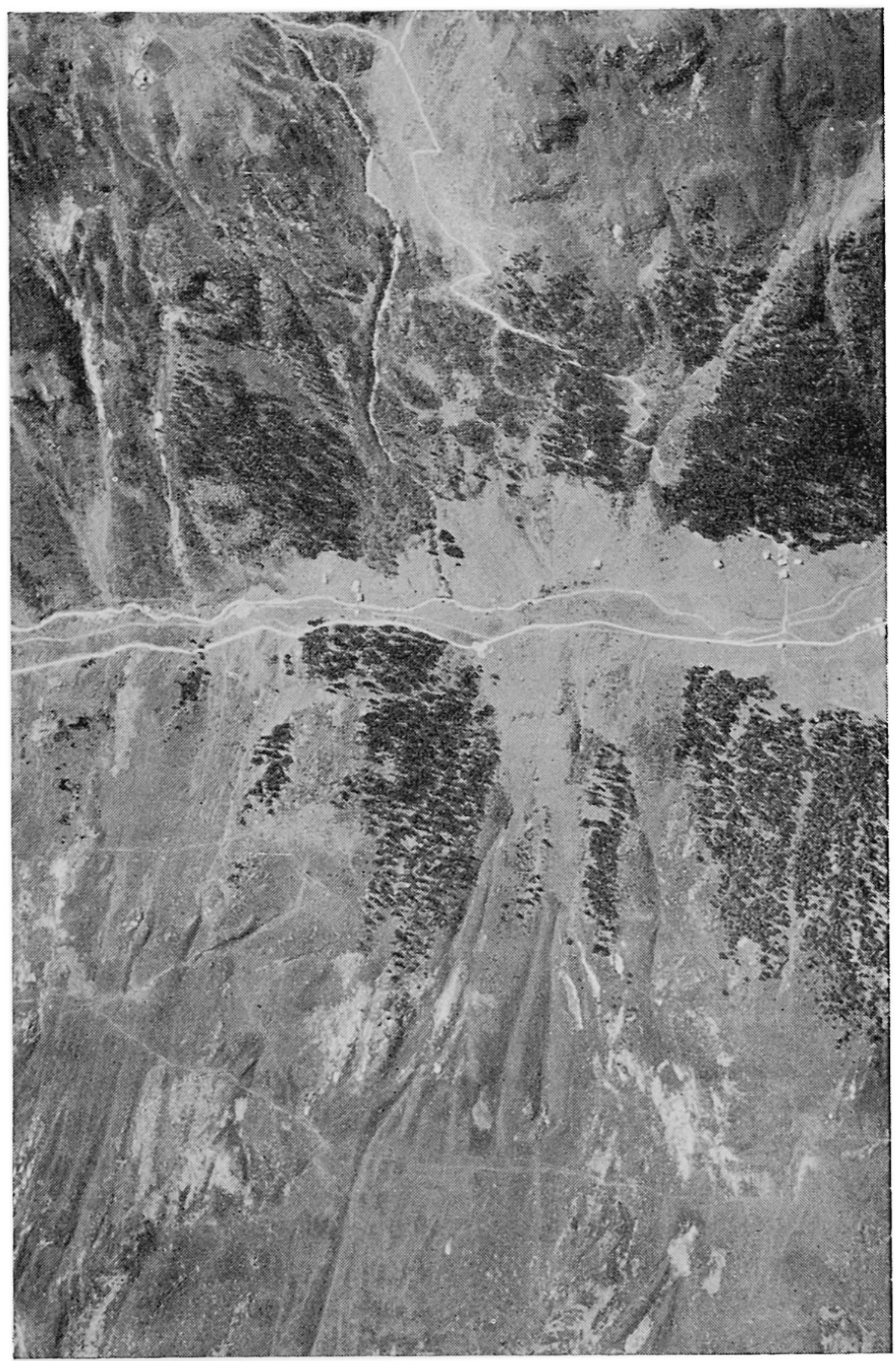

Abb. 1:

Luftbild (August

1930), Maßstab

ungefähr

$1: 16000$, zeigt

das Ende

des Waldes im

Dischma, mit dem

hintersten dau-

ernd bewohnten

Haus (Gulerigen-

hus, ganz rechts)

sowie die Alp

Boden-Rüedisch-

tälli und die Alp

Chintschhus.

(Der Talausgang

liegt immer

rechts.)

2. Der Herbst (September), zur Zeit der Laubverfärbung, mit ebenfalls guten Grautonabstufungen und dem Überblick über die Art der Landnutzung (gemähte und beweidete Wiesen usw.).

Nachteilig wirken sich zu diesen Jahreszeiten die relativ tief stehende Sonne (lange Schatten) und evtl. Schneebedeckung (Altschneeflecken im Vorsommer, früher Schneefall im Herbst) aus. Gleichwohl sind diese Luftbilder Sommeraufnahmen vorzuziehen, wenn sie zur Zeit des relativ höchsten Sonnenstandes, also über Mittag und unter Berücksichtigung der topographischen Verhältnisse, geflogen wurden. Sommerbilder enthalten viel geringere Grautonkontraste und erlauben keinen Gesamtüberblick, z. B. über die gemähten Wiesen. 


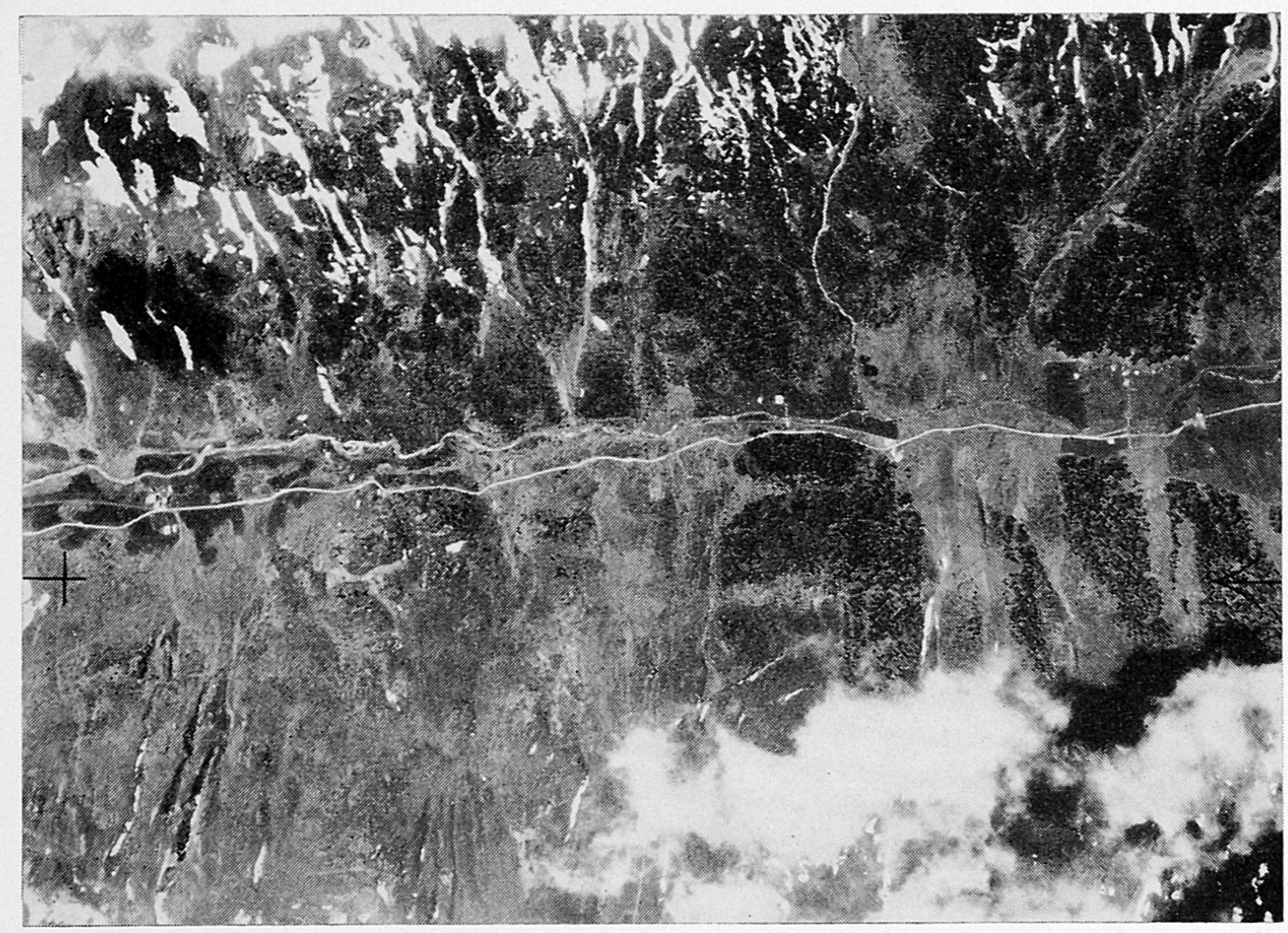

Abb. 2: Luftbildausschnitt (31. Mai 1960, Maßstab ungefähr 1:21000) mit Gulerigenhus (ganz rechts), Alp Chintschhus (Mitte) und Alp Gadmen (links).

Weit mehr als das Einzelbild vermag nun der Vergleich von Bildserien vom gleichen Gebiet, die zeitlich mehrere Jahre auseinander liegen, den Werdegang der Landschaftsentwicklung aufzudecken. Verschiedene Zustände werden für einen bestimmten Zeitpunkt erfaßt und ermöglichen die Ermittlung der Veränderungen und deren chronologische Einordnung.

Die zahlenmäßige Erfassung der räumlichen Veränderungen erfordert die Übertragung der im Luftbild erkannten und ausgeschiedenen Einheiten in die topographische Karte. Luftbild- und Kartenmaßstab sollten dabei nicht sehr verschieden sein. In der Karte können dann die einzelnen Flächen planimetriert und ihre Größe berechnet werden.

Die Übertragung Luftbild - Karte kann auf verschiedenem Weg vorgenommen werden.

1. Unter Verwendung von photogrammetrischen Präzisionsinstrumenten (1. und 2. Ordnung), wie Autograph, Stereoplanigraph usw.

2. Übertragung mit Stereokartiergeräten einfacher Art mit Näherungslösung, wie Stereotop, Radial Line Plotter usw.

3. Mit Hilfe von Geländepunkten, die in der Karte und im Luftbild (im Stereomodell) eindeutig bestimmbar sind und mit Interpolation dazwischen. Großmaßstäbige Isohypsenkarten mit kleiner Aequidistanz und genügend derartigen Fixpunkten sind dafür unerläßlich. Da in unserem Arbeitsgebiet eine solche Karte zur Verfügung stand (1:10 000, $10 \mathrm{~m}$ Aequidistanz), erfolgte die Übertragung nach der letztgenannten Methode. 


\section{DAS LUFTBILD- UND KARTENMATERIAL}

Die sinnvolle Lösung derartiger Aufgaben setzt die Durchführung von sorgfältig geplanten Bildflügen voraus, wobei vor allem Maßstab, Jahres- und Tageszeit, Phänologie, Sonnenstand und die topographischen Verhältnisse berücksichtigt werden müssen. Für die nachstehenden Untersuchungen im Dischmatal bei Davos standen zahlreiche Bildflüge, aufgenommen vom Luftaufklärungsdienst Dübendorf, aus dem Zeitraum von 1956-1962 zur Verfügung. Diese Aufnahmen wurden im Zusammenhang mit dem großangelegten Aufforstungsversuch auf der Stillbergalp im Dischma der Eidgenössischen Anstalt für das forstliche Versuchswesen, Birmensdorf, verbunden mit Schneeund Lawinenuntersuchungen des Eidg. Instituts für Schnee- und Lawinenforschung, Weißfluhjoch, Davos, geflogen. Zudem gibt es vom Dischmatal einen alten Bildflug aus dem Jahr 1930, der bezüglich Qualität und Maßstab recht erfreulich ist und daher vergleichende Betrachtungen über eine längere Zeitperiode gestattet. Für diese Arbeit wurden die folgenden Bildserien verwendet:

\begin{tabular}{|c|c|c|c|c|}
\hline Kamera & 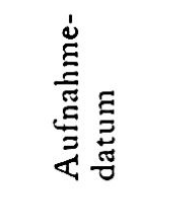 & 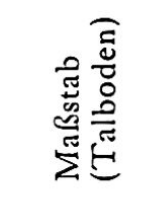 & 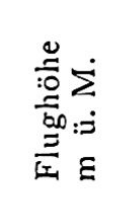 & \\
\hline $\begin{array}{l}\text { Zeiss Doppelkammer, Konvergent- } \\
\text { aufnahmen, Glasplatten. } \mathrm{f}=210 \mathrm{~mm}\end{array}$ & $\begin{array}{l}\text { Aug. (?) } \\
1930\end{array}$ & $\begin{array}{l}\text { ungefähr } \\
1: 16000\end{array}$ & 5200 & $\begin{array}{l}\text { Eidg. Landes- } \\
\text { topographie }\end{array}$ \\
\hline $\mathrm{K}-24, \mathrm{f}=178 \mathrm{~mm}$ & 15. 9. 56 & $1: 15000$ & 4300 & $\begin{array}{l}\text { Luflauf- } \\
\text { klärungsdienst } \\
\text { Dübendorf }\end{array}$ \\
\hline dito & 31.5 .60 & $1: 21000$ & 5400 & dito \\
\hline dito & 9. 9.60 & $1: 44000$ & 9500 & dito \\
\hline dito & 23.9 .60 & $1: 21000$ & 5400 & dito \\
\hline
\end{tabular}

Als Kartenunterlage diente der «Übersichtsplan der Landschaft Davos» 1:10ooo, Blätter Scaletta- und Flüelapa B (Schw. Grundbuchvermessung). Daneben können auch die Blätter der LK $1: 25$ ooo, 1197 Davos und 1217 Scalettapa $\beta$ verglichen werden.

Für die Überlassung von Karten- und Photomaterial möchte ich der Eidg. Anstalt für das forstliche Versuchswesen (Direktor Prof. Dr. A. Kurth) und dem Luftaufklärungsdienst Dübendorf (Herrn Oberst Loepfe) für die Aufnahme mehrerer Bildflüge an dieser Stelle bestens danken.

\section{DIE ALPWIRTSCHAFT DES DISCHMATALES}

Die Alpwirtschaft der Landschaft Davos wurde von Senn (1950, 1952) eingehend untersucht und beschrieben. Sie zeichnet sich durch Einzelhofsiedlungen (Walser) mit meist zweistufigem Alpbetrieb (Talgut - Alp) aus. Die Maiensäß-Stufe fällt weg. Die Alpen sind meistens in Privatbesitz und werden von einem oder wenigen Bauern gemeinsam bewirtschaftet.

Im Dischma, dem ca. $15 \mathrm{~km}$ langen Seitental, sind die Betriebsstufen einerseits übereinander am Hang und andererseits hintereinander in der Talsohle angeordnet. Soweit die Hänge bewaldet sind (der Wald endet nach ca. dem ersten Drittel wie abgeschnitten), liegen auf der sonnenexponierten Seite des Talbodens die einzelnen Höfe inmitten der saftigen Fettwiesen. Am Rande des Wiesenareals gegen den Wald stehen die Außenställe, von denen aus das Vieh im Frühsommer und Herbst auf die Waldweide getrieben wird. Auf den Verflachungen an oder über der Waldgrenze liegen die Alphütten. Die Distanzen zwischen den einzelnen Betriebsstufen sind also gering. Jede Alp besteht aus mehreren Gebäuden, in deren nächster Umgebung sich immer ein Wiesenstück (Alpfettwiese) befindet. Bei den in den Hängen liegenden Alpen kann es sich auf eine kleine ummauerte Fläche beschränken. Im hinteren Talteil, in der oft ziemlich weiten und flachen Sohle, kann es entsprechend umfangreicher sein. Stets umgrenzen solide Steinmäuerchen die Fettwiesen, und Viehtriebe führen zwischen diesen hindurch. Auch die höchstgelegenen Hangpartien werden vom Talboden aus bestoßen. Praktisch alle nicht gemähten Flächen

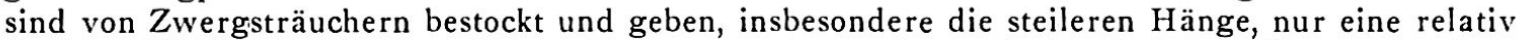
dürftige Weide ab. Die eigentlichen Alpmatten nehmen einen sehr beschränkten Raum ein, oberhalb ca. 2200-2300 m ü. M., worauf sehr schnell die steilen Gipfelpartien mit anstehendem Fels und den ausgedehnten, praktisch vegetationsfreien Blockschuttfeldern einsetzen. 


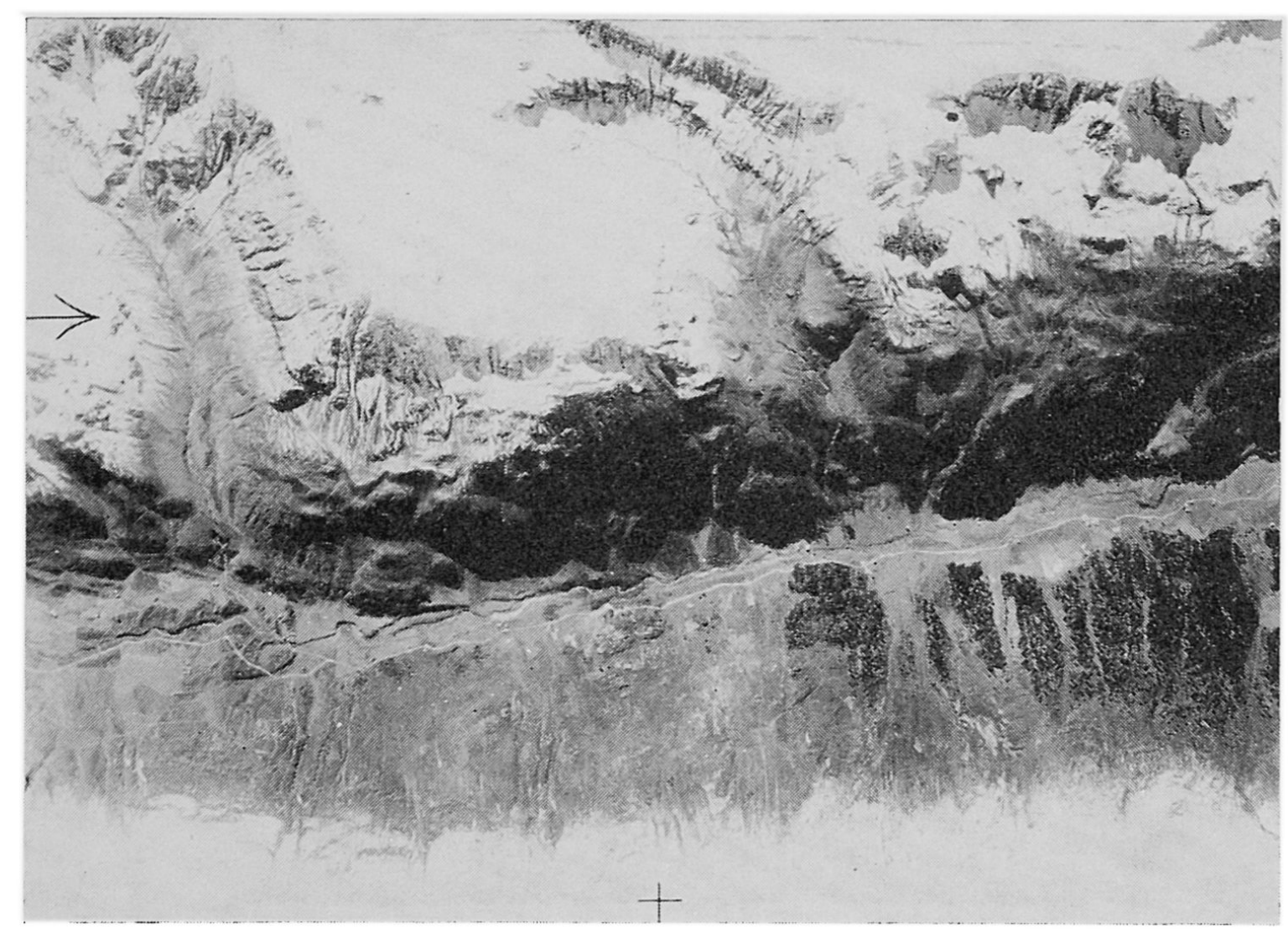

Abb. 3: Der Luftbildausschnitt (9. September 1960, Maßstab ungefähr 1:44000) zeigt das Tal von Inner Hof (ganz rechts) über Teufi, Gulerigenhus, Alp Chintschhus, Alp Gadmen, Alp Am Rin bis zur Jenatschalp (ganz links). Von $S W$ münden zwei kleine Hängetäler, Rüedischtälli (rechts) und Rinertälli.

\section{DAS UNTERSUCHUNGSBEISPIEL}

Aus der Vielfalt der aufgezeigten Möglichkeiten sollen hier die Veränderungen im Mähwiesenareal eingehend untersucht und dargestellt werden. Der Strukturwandel in der Alpwirtschaft, d. h. das Ausmaß der Extensivierungsvorgänge, kommt hier wohl am deutlichsten zum Ausdruck und bietet für die Luftbildinterpretation gleichzeitig die interessantesten und vielschichtigsten Probleme. Das Erkennen anderer Veränderungen, z. B. im Wegnetz, Wüstlegung oder Neubau von Gebäuden usw. dagegen bereitet viel weniger Mühe.

Gebietsmäßig wurde der bearbeitete Raum auf die im Talboden liegenden Alpen eingeschränkt, die mit Ende des Waldes einsetzen und vom hintersten dauernd bewohnten Haus, Gulerigenhus (1700 m. ü. M.) bis Dürrboden (2000 m. ü. M.) reichen.

Im Luftbild können alle Flächen, die irgendwann einmal in eine Wiese umgewandelt und gemäht wurden, identifiziert und abgegrenzt werden, auch wenn sie heute längst wieder aufgegeben wurden und sich in eine $Z$ wergstrauchweide zurückverwandelt haben (Haefner 1963b). Als Abgrenzungskriterien können hiefür benutzt werden :

1. Bestehende Wiesen, von Mäuerchen umgeben, die heute noch gemäht werden.

2. Ummauertes Wiesenareal, das nicht mehr geschnitten wird und die typischen Weidetexturen aufzeigt.

3. Weideflächen, die deutlich von Mäuerchen oder Mauerresten umgrenzt sind und deren $Z$ wergstrauchbedeckung sich speziell aufgrund ihres lockeren Bestandes von der Umgebung abhebt. 
4. Flächen, die sich in der Zwergstrauchbedeckung nicht mehr von der Umgebung unterscheiden, die aber mit Hilfe von mehr oder weniger gut erhaltenen Mauerresten als einmal zum Wiesenareal gehörend ausgeschieden werden können.

Der Umstand, daß alle Wiesen, wenn sie nicht an einen Bach grenzen, mit schützenden Mäuerchen umgeben wurden, die man einzig zu Schutzzwecken gegen das weidende Vieh errichtet hat, ermöglicht in ersier Linie eine umfassende Ausscheidung aller einmal gemähten Gebiete.

Gleichzeitig legen sie auch die exakte Grenze zwischen Wiese und Weide fest. Mäuerchen werden zudem bei der Aufgabe einer Wiese überhaupt nicht oder nur wenig zerstört und bleiben, mindestens bruchstückweise, sehr lange erhalten. Sie können im Luftbild bei stereoskopischer Betrachtung bei Maßstäben bis ca. 1:18 000 eindeutig erkannt und ausgeschieden werden. Weitere Anhaltspunkte ergeben sich aus der Vegetationsbedeckung, den Hauswüstungen (immer innerhalb oder am Rande einer Wiese gelegen) und dem Wegnetz.

Zusätzlich vermitteln uns die verschiedenen Arten der Vegetationsbedeckung, speziell der Grad der Wiederbewachsung mit Zwergsträuchern, eine chronologische Abfolge des Zeitpunkts der Aufgabe der Wiese. Je weiter dieser zurückliegt, desto dichter ist die Bestockung mit $Z$ wergsträuchern. Über den absoluten Zeitpunkt der Aufgabe dagegen kann auf diesem Wege nichts ausgesagt werden. Hier müßten Luftaufnahmen aus früheren Jahren helfen, die allerdings auch in den günstigsten Fällen zu wenig weit zurückreichen.

Die Ausscheidung des gesamten, einmal gemähten Wiesenareals wurde mit Hilfe der Luftbilder von 1956 vorgenommen und nach der vorher beschriebenen Methode in die Karte 1:10 000 übertragen. In allen drei Karten ist dieses Wiesland eingetragen. Es vermag am eindrücklichsten zu zeigen, wie stark sich der Rückgang ausgewirkt hat. Immerhin muß gesagt werden, daß aus dem Luftbild nicht hervorgeht, da $\beta$ dieses Gesamtareal wirklich einmal in seinem maximalen Umfang gemäht wurde. Es können Wiesen bereits wieder aufgegeben worden sein, während noch neue hinzukamen. Der Beleg der Gleichzeitigkeit kann aus dem Luftbild nicht erbracht werden. Ebenso kann nicht mehr in einzelne Wiesentypen unterschieden werden.

Diesem zeitlich nicht faßbaren Gesamtareal der Mähwiesen wurden nun Zustände, die zeitlich genau belegt sind, gegenübergestellt und so die Wandlungen und der zeitliche Ablauf der Entwicklung anschaulich gemacht.

\section{Karte I: Gemähte Wiesen r930}

Das genaue Datum des Fluges von 1930 läßt sich leider nicht mehr ermitteln. Am Stand der landwirtschaftlichen Arbeiten (alle Heuberge sind z. B. geschnitten) kann jedoch abgeleitet werden, daß die Aufnahmen in den letzten Augusttagen, evtl. anfangs September, gemacht worden sein müssen. Die Bilder eignen sich nach Maßstab und Qualität vorzüglich zur Ausscheidung der gewünschten Einheiten (Abb. 1).

Legende zu den Karten auf Seite 225

\begin{tabular}{|c|c|c|c|c|c|}
\hline$\left[\begin{array}{l}0 \% \\
0000\end{array}\right.$ & Wald & & $\begin{array}{l}\text { Ehemaliges } \\
\text { Wiesenareal }\end{array}$ & 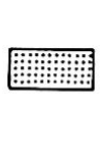 & $\begin{array}{l}\text { Talfettwiese } \\
\text { (nicht mehr in die } \\
\text { Betrachtung } \\
\text { miteinbezogen) }\end{array}$ \\
\hline 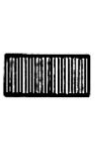 & Wiese, geschnitten & 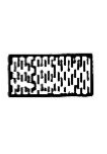 & $\begin{array}{l}\text { Wiese, } \\
\text { die wahrscheinlich } \\
\text { noch geschnitten } \\
\text { wird }\end{array}$ & 焉: & $\begin{array}{l}\text { Fettwiese } \\
\text { Weitere Erklärungen im Text }\end{array}$ \\
\hline
\end{tabular}




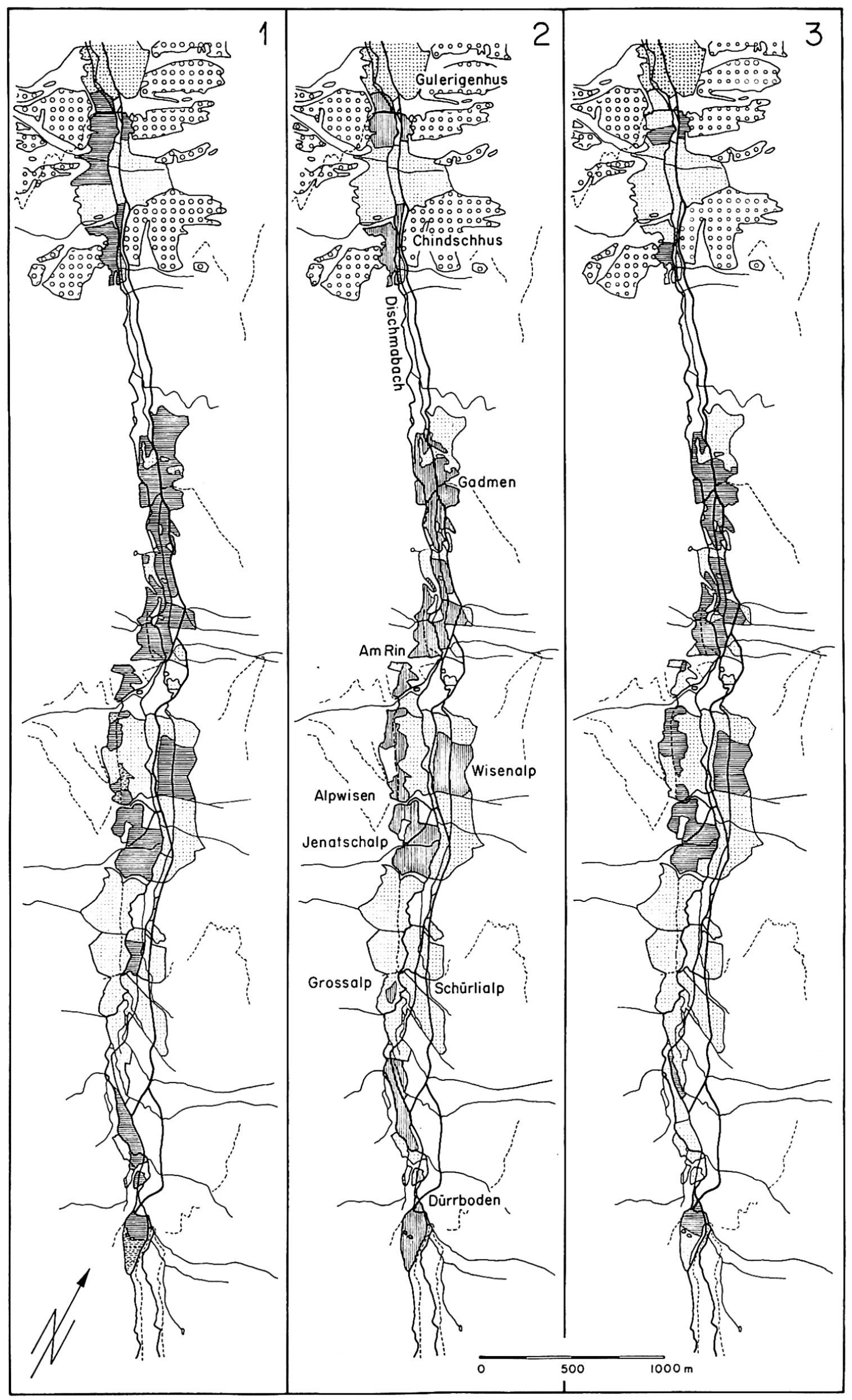


Gemähte Wiesen sind leicht zu erkennen an ihrem hellen Grauton, der über die ganze Fläche regelmäßig und einheitlich bleibt, an der gleichmäßigen, glatten Oberfläche, den scharfen Rändern und dem Fehlen jeglichen Stereoeffekts. Mähder weisen eine typische Längsstreifung (Mähen im Zweijahres-Rhythmus) auf. Weitere Anhaltspunkte können Mähstreifen, Heuschochen und ähnliches mehr ergeben.

Obwohl der Stand der landwirtschaftlichen Arbeiten recht weit fortgeschritten ist, kann bei diesem Flug nicht mit Sicherheit gesagt werden, ob wirklich alle in diesem Jahr geschnittenen Wiesen erfaßt sind. Die Aufnahmezeit liegt etwas zu früh. So fallen einige Wiesenstücke speziell auf durch ihren kompakten, dunklen Grauton mit leichtem Stereoeffekt, was auf hohen, satten Graswuchs schließen läßt. Daher ist zu vermuten, daß diese besonders ertragreichen Wiesen noch gemäht werden, da sie sonst zumindest als Weide benutzt worden wären. Es handelt sich allerdings nur um kleine Abschnitte. Sie wurden mit einer eigenen Signatur gekennzeichnet.

Karte 3: Gemälte Wiesen I960

Im Vergleich dazu stellt Karte 3 den Umfang der Mähwiesen 30 Jahre später dar. Hier konnten nun mit einem späten Septemberflug alle geschnittenen Flächen sicher erfaßt werden (Abb. 4). Gelegentlich waren die Interpretationsmerkmale infolge des leichten Nachwachsens des Grases und dem Fehlen der typischen Mähtexturen bereits wieder etwas verwischt. Daher wurde noch eine Bildserie von anfangs September des gleichen Jahres hinzugezogen, die zwar im Maßstab kleiner und viel ungünstiger war, in gewissen Fällen aber eindeutigere Aussagen ermöglichte. (Abb. 3). Der Unterschied in der Gesamtfläche ist bedeutend. Sie hat sich erheblich vermindert.

\section{Karte 2: Fett-wiesenareal 1960}

Fettwiesen, d.h. Wiesen, die regelmäßig gedüngt werden und daher wesentlich höhere Erträge geben, zeichnen sich gegenüber den Magerwiesen und Weiden durch ein früheres Einsetzen des Graswuchses aus. Im Frühsommer weisen sie bereits einen geschlossenen Rasenteppich von sattem Grün auf, während die Umgebung noch in bräunlich-gelben Farbtönen verharrt. Im Luftbild treten daher die Fettwiesen ebenfalls in einem kompakten, dunklen Grauton hervor und können leicht erkannt werden (Abb. 2). Das Areal der Fettwiesen zeigt uns die am besten gepflegten und am intensivsten genutzten Wirtschaftsflächen an, darf aber nicht mit dem Areal der gemähten Wiesen gleichgesetzt werden. Vielmehr können auch Fettwiesen aufgegeben und nur noch als Weide benutzt werden. Auch wenn sie nicht mehr gedüngt werden, können sie sich noch recht lange als Fettwiesen manifestieren, allerdings allmählich in abgeschwächtem Maße.

Der Umfang der Fettwiesen im Jahre 1930 kann nicht bestimmt werden, da aus jenem Jahr kein entsprechender Bildflug vorliegt. Es ist aber anzunehmen, daß er kaum wesentlich von demjenigen von 1960 differiert.

\section{DIE INTERPRETATION DER KARTEN}

Obwohl die drei Karten eigentlich für sich selbst sprechen, sei doch auf einige besondere Merkmale hingewiesen. Zuerst fällt der bedeutende Rückgang der Mähwiesen auf, der, wie der Flug von 1930 beweist, zeitlich schon weit zurückreichen muß. In der jüngsten Vergangenheit wurden vor allem die topographisch ungünstiger gelegenen $\mathrm{Ab}$ schnitte aufgegeben. Der gemähte Teil schrumpft immer mehr auf die unmittelbare Umgebung der Alphütten zusammen. Besonders auffallend ist dies bei den vordersten Alpen im Talboden (Boden und Chintschhus). Es können aber auch ganze Alpen aufgegeben werden, wie z. B. zwischen Jenatschalp und Dürrboden. Hier finden sich dann 


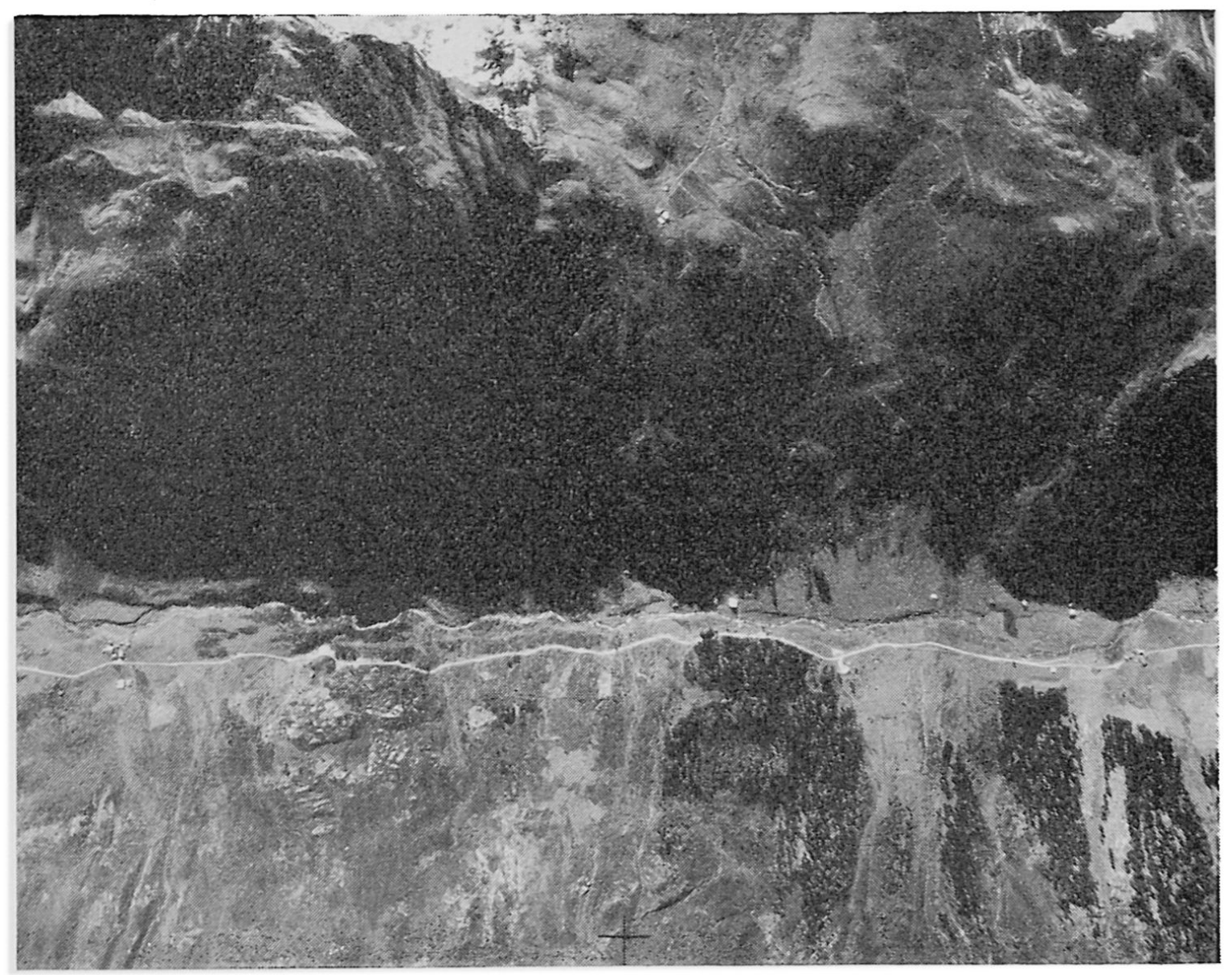

Abb. 4: Der Luftbildausschnitt (23. September 1960, Maßstab ungefähr 1:21000) gibt das Gebiet zwischen Gulerigenhus und Alp Gadmen wieder. Es machen sich bereits störende Bergschatten bemerkbar.

auch immer Hauswüstungen, wie z. B. ein ganzes Alpdörfchen auf einer Moräne südlich der Jenatschalp (Pt. 1944.7, LK 1: 25 000).

Interessant ist vor allem der Vergleich zwischen dem besten Wiesland, demjenigen der Fettwiesen und dem restlichen Anteil. 1930 reichte der geschnittene Teil noch erheblich über das Fettwiesenareal hinaus und erfaßte auch relativ ungünstig gelegene Magerwiesen, z. B. an steilen Hängen. Inzwischen sind alle Magerwiesen aufgegeben worden, und auch von den Fettwiesen werden nur noch Teile gemäht. Seit 1960 hat sich dieser Rückgang sogar noch verstärkt. Zahlreiche Fettwiesen wurden mit Hilfe von elektrischen Zäunen unterteilt und werden, mindestens größtenteils, nur noch beweidet.

\section{SCHLUSSBEMERKUNG}

Das Luftbild ist wie kein zweites Hilfsmittel geeignet, uns genauen Aufschluß über die räumlichen Veränderungen der Kulturlandschaft in ihrer zeitlichen Abfolge zu geben. Alle Bildflüge, insbesondere solche älteren Datums, sollen daher, auch wenn sie hinsichtlich der Qualität nicht mehr allen Ansprüchen genügen, sorgfältig registriert und aufbewahrt werden. Das hier in Bezug auf die Alpwirtschaft aufgezeigte Bild ist leider wenig erfreulich und mahnt zum Aufsehen und zu umgehenden, tiefgreifenden Reform-Maßnahmen. 


\section{LITERATURVERZEICHNIS}

Chabrol, P.: Quelques emplois de la photographie aérienne dans un service de restauration des terrains en montagne. Revue Forestière Française, No. 11, 1953, Nancy, p. 767-773.. - Einevoll, O.: Luftbilete ved fjellbeitegransking (Air-photos in mountain region grassland surveying). Tidsskirft for det norske landbruk, 65, Oslo, 1958, p. 101-134. - Gutersohn, H. : Neuere Wandlungen der Schweizer Kulturlandschaft. Geogr. Rundschau, 15. Jg., Nr. 1, p. 1-6, 1963; der gegenwärtige Wandel alpiner Kulturlandschaften. Geographica Helvetica 19, 196+, S.138-14o. - Haefner, H.: Vegetationskarten des Dischmatales. Manuskript, Geogr. Institut der Universität Zürich 1963; Vegetation und Wirtschaft der oberen subalpinen und alpinen Stufe im Luftbild. Landeskundliche Luftbildauswertung im mitteleuropäischen Raum, Heft 6, Bundesanstalt für Landeskunde und Raumforschung, Bad Godesberg 1963, 117 p. - Kuoch, R: Ausaperung im Luftbild, - Zweck und Methode einer Auswertung im Dischmatal. In: Die Anwendung des Luftbildes im schweizerischen Forstwesen, Mitteilungen der Schweiz. Anstalt für das forstliche Versuchswesen, Bd. 38, Heft 1, p.187-191, Birmensdorf 1962. - Paschinger, H.: Strukturwandlungen im Bergbauerntum. Hermann von Wissmann-Festschrift, p. 357-362, Tübingen 1962. — Ramser, E.: Der Alpkataster als Planungsgrundlage für die Berggebiete. In: Güter-, Rebberg- und Waldzusammenlegung, Baulandumlegung, Landesplanung. Ergänzter Sonderdruck aus: Plan Vol. 8, Nr. 3, p. 21-2t, 1951. - Rey, P.: Photographie aérienne et connaissance du milieu alpin. Etude botanique de l'étage alpin, particulièrement en France, Bayeux 195t, (Congrès Int. de Botanie), p. 23-26. - Rhody, B.: Die Flugaufnahme als Zeitdokument, an einem Beispiel aus dem Gebirgswald dargestellt. In: Die Anwendung des Luftbildes im schweizerischen Forstwesen, Mitteilungen der Schweiz. Anstalt für das forstliche Versuchswesen, Bd. 38, Heft 1, p. 193-199, Birmensdorf 1962. - Senn, U.: Davos - eine geographische Auswertung von Nutzungs- und Siedlungskarte mit Protokoll, unter besonderer Berücksichtigung der Alpwirtschaft. Diplomarbeit (Manuskript), Geogr. Institut der Universität Zürich, 1950; Die Alpwirtschaft der Landschaft Davos. Geographica Helvetica, Vol. 7, Heft 4, p. 265-350, 1952. - Splechtner, F.: Die Bedeutung alpwirtschaftlicher Luftbildforschung für das gesamte Alpverbesserungswesen. Internationaler Holzmarkt, Heft 8, Wien, 1945; Die Alpwirtschaftslandschaft von Kolm-Saigurn - Ein Versuch zur Eintührung der Luftbilderkundung in der alpwirtschaftlichen Forschung. Abhandlungen der Geographischen Gesellschaft in Wien, Vol. 16, Heft 2, p. 4-to, 1948. - Steiner, D.: Die Verwendung von Luftbildern bei wirtschaftsgeographischen Untersuchungen im alpinen Gebiet. Diplomarbeit (Manuskript), Geogr. Institut der Universität Zürich, 1956; Die Bedeutung der jahreszeitlichen Aspekte für die Landnutzungsinterpretation auf Luftbildern, gezeigt am Beispiel einer alpinen Agrarlandschaft (Heinzenberg, Graubünden). Landeskundliche Luftbildauswertung im mitteleuropäischen Raum, Heft 3, p. 51-55, Bundesanstalt für Landeskunde und Raumforschung, Bad Godesberg, 1960. - Steiner, D. und Haefner, H.: Die Verwendung von Luftbildern als Planungsunterlage für die wirtschaftliche Neuordnung im Gebirge. Transactions of the Symposium on Photo Interpretation, Delft 1962, Archives Intern. de Photogrammétrie, Vol. XIV, p. 338-347, Delft 1963. - Suter, K.: Der Zerfall des alpinen Nomadismus im Wallis und Tessin. Mitteilungen des Österreichischen Geographischen Gesellschaft, Bd. 105, Heft I/II, p. 180-187, 1963.

\section{LES CHANGEMENTS DANS L'ÉCONOMIE ALPESTRE VUE PAR LA PHOTOGRAPHIE AÉRIENNE}

Ces dernières années les altérations dans l'économie alpestre et surtout le procès d'extension ont été considérables. A l'aide des photographies aériennes prises à plusieurs intervales, il est possible de reconstruire les phases de ce procès à travers le temps. La photo aérienne relève toujours les conditions à un moment exact aussi bien que des éléments historiques et en conséquence permet un projettement sensé. Les saisons les plus favorables pour la photographie aérienne en haute montagne sont le printemps (mai-juin, selon l'altitude) et l'automne (septembre). Pour une évaluation exacte de surfaces, les extraits doivent être transcrits sur la carte et puis planés.

Les possibilités et méthodes de l'interprétation des photographies aériennes sont démontrées par une exemple du «Dischmatal» près Davos (Grisons). C'est la transformation des prairies qui est examinée dans ce cas. Toutes les prairies une fois fouchées peuvent être vérifiées à l'aide des clôtures même si aujourd'hui elles sont couvertes de landes. Ces régions sont indiquées dans les trois cartes. Carte no. 1 et 3 montrent les prairies fouchées en 193o et puis en 1960. La diminution est évidente. En 1960 même les prairies de première qualité (prairies grasses) (carte no. 2) n'ont été toutes fouchées. 